\title{
La sentence de Yhwh comme chemin de rédemption et deuxième possibilité de conversion. Analyse rhétorique de Gn 3
}

\author{
Germano Lori et Francesco Giosuè Voltaggio \\ Traducteur : Roland Meynet
}

\section{(2) OpenEdition}

Journals

Édition électronique

URL : https://journals.openedition.org/rhetorique/497

DOI : 10.4000/rhetorique.497

ISSN : 2270-6909

Éditeur

UGA Éditions/Université Grenoble Alpes

Édition imprimée

ISBN : 978-2-84310-343-8

\section{Référence électronique}

Germano Lori et Francesco Giosuè Voltaggio, « La sentence de Yhwh comme chemin de rédemption et deuxième possibilité de conversion. Analyse rhétorique de Gn 3 », Exercices de rhétorique [En ligne], 8 | 2017, mis en ligne le 25 janvier 2017, consulté le 29 novembre 2021. URL : http:// journals.openedition.org/rhetorique/497 ; DOI : https://doi.org/10.4000/rhetorique.497

Ce document a été généré automatiquement le 29 novembre 2021.

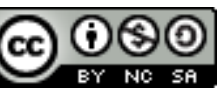

Les contenus de la revue Exercices de rhétorique sont mis à disposition selon les termes de la Licence Creative Commons Attribution - Pas d'Utilisation Commerciale - Partage dans les Mêmes Conditions 4.0 International. 


\title{
La sentence de Yhwh comme chemin de rédemption et deuxième possibilité de conversion. Analyse rhétorique de Gn 3
}

\author{
Germano Lori et Francesco Giosuè Voltaggio \\ Traduction : Roland Meynet
}

\section{NOTE DE L'ÉDITEUR}

Pour agrandir les images, cliquer en-dessous sur les mentions « Agrandir » (taille moyenne) ou «Original »(taille réelle).

$1 \quad$ L'objectif de ces pages est de montrer les résultats de l'analyse rhétorique biblique et sémitique ${ }^{1}$ appliquée à la séquence de $\mathrm{Gn} 3$. Ce chapitre comprend cinq passages qui ont été analysés séparément dans des contributions antérieures ${ }^{2}$. Le premier (1-5) présente le dialogue entre le serpent et la femme, le second (6-8) la faute d'Adam et d'Ève. Le troisième expose le rîb (« controverse ", « dispute », " procès ») de Dieu envers l'homme et la femme; Dieu dévoile leur péché, mais ils refusent de reconnaître leur propre responsabilité. Le quatrième passage (14-19) montre les conséquences de la transgression et du refus de reconnaître la responsabilité, qui retombent, dans l'ordre, sur le serpent, sur la femme et sur l'homme; chaque phrase est une parole de Dieu ("dit »: 14.16.17), mais, au moment même où il la prononce, il annonce aussi une promesse de salut pour l'homme (15). Le dernier passage (20-24) enfin présente l'expulsion de l'homme et de la femme du jardin d'Éden. Les passages se correspondent en miroir, et le troisième, au centre, fournit la clé de l'interprétation de l'ensemble. Le 
refus de reconnaître leurs propres fautes est la clé de l'expulsion de l'Éden. La séquence peut être synthétisée par le schéma suivant ${ }^{3}$ :

\begin{tabular}{|c|c|}
\hline La tentation du serpent & $3,1-5$ \\
\hline La faute d'Adam et Ève & $3,6-8$ \\
\hline LE JUGEMENT DE DIEU & $3,9-13$ \\
\hline La sentence de Dieu & $3,14-19$ \\
\hline L'expulsion de l'Éden & $3,20-24$ \\
\hline
\end{tabular}

\section{Les passages extrêmes $(3,1-5 ; 20-24)$}

\section{Composition}

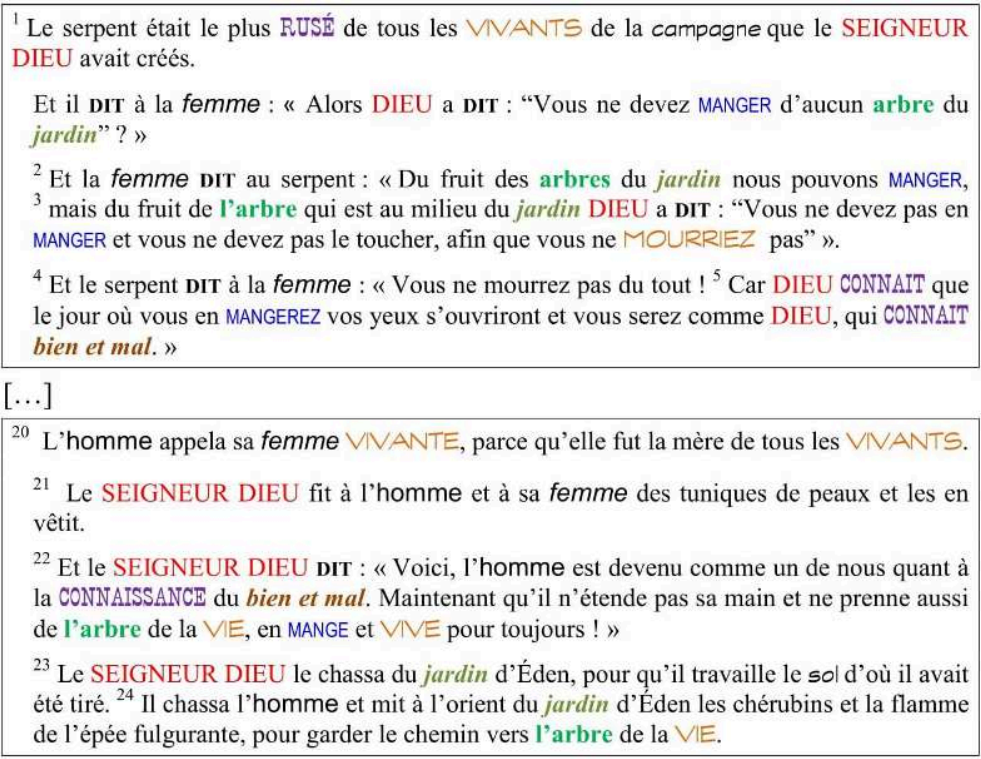

2 Le premier passage (1.5) comprend deux parties. La première (1a) est composée d'un segment qui joue le rôle de courte introduction narrative. La deuxième (1b-5) est constituée par trois sous-parties qui forment une structure concentrique $(1 \mathrm{~b} ; 2-3$ et 4-5). C'est le dialogue entre le serpent et la femme scandé par « et il a dit », qui apparaît au début de chaque sous-partie.

3 La dernier passage (20-24) comporte aussi deux parties. Là aussi, la première (20) est composée d'un segment qui joue le rôle de courte introduction. La deuxième partie (21-24) est composée de trois sous-parties, dont chacune commence par l'expression "le Seigneur Dieu » (21, 22 et 23-24). Les sous-parties extrêmes sont de récit, tandis que la sous-partie centrale rapporte les paroles de Yhwh. Il s'agit encore d'une structure concentrique, comme dans le premier passage. La parole de Yhwh est tout à fait énigmatique, ce qui est souvent le cas au centre des constructions concentriques. Enfin on notera la répétition des expressions " chassa l'homme " / "le chassa » et " jardin d'Éden » dans les deux derniers versets (23-24) du passage, qui sont aussi les derniers versets de la séquence.

4 En outre, les passages extrêmes se correspondent globalement. Le premier (1-5) est le préambule de l'histoire du péché originel, avec la scène de la femme tentée par le 
serpent dans le jardin ; le dernier (20-24) est la conclusion de la scène avec l'expulsion hors du jardin.

5 Le mot «Dieu » est le plus fréquent dans les deux passages, il apparaît huit fois (1bis. 3.5bis.21.22.23), dont quatre accompagné du tétragramme sacré "Yhwh», pour composer l'expression « le Seigneur Dieu ». Cette expression se trouve toujours dans la bouche du narrateur, aux extrémités (1.23) de l'ensemble du chapitre (en termes extrêmes) et au début des deux étapes ( 1.20 ; en termes initiaux). Le mot "vivant(s)» joue le même rôle (1.20bis) et rappelle les mots «vie» / "vivante» (22-23), qui sont opposés au verbe «mourir» de $2^{4}$. Le substantif «femme ${ }^{5}$ » $(1.2 .4 ; 20-21)$ joue aussi le rôle de termes initiaux (1.20). Les mots « jardin ${ }^{6}$ », « arbre » et " campagne » / « sol » de 1 et 23 peuvent être considérés comme termes extrêmes de la séquence entière. Le substantif «arbre » $(2-3 ; 22)$ et le verbe « manger $^{7} »(2-3 ; 22)$ apparaissent au centre des passages respectifs et peuvent donc être considérés comme des termes centraux. On notera aussi les correspondances lexicales suivantes :

• «dire » $(1 b i s ; 2 b i s ; 4.22)$;

- les expressions où se retrouvent les termes « connaître » / « connaissance », «bien » et « mal » (5.22) auxquels pourrait être aussi associé le terme « rusé » (1).

\section{Interprétation}

6 En comparant les passages extrêmes, la première chose qui saute aux yeux est que l'arbre dont il est interdit à l'homme de manger au début du récit est celui de la connaissance du bien et du mal (Gn 2,9 et 3,2), tandis que, dans le passage final, l'arbre dont il ne pourra pas s'approcher est celui de la vie (22-24). En outre, suivant les indications du commandement que Dieu donne à Adam (16-17), il est clair qu'il pouvait manger de tous les arbres du jardin, y compris donc celui de la vie. Cependant, la chose la plus surprenante n'est pas qu'il lui soit interdit, après le péché, de se nourrir de l'arbre de la vie, étant donné que Dieu l'avait averti de la conséquence que cela aurait entraîné : « Le jour où vous en mangerez, certainement vous mourrez » (17). La chose la plus étrange au contraire est que désormais l'accès de l'arbre de la connaissance du bien et du mal ne soit pas interdit à Adam, comme si cela voulait dire qu'il peut encore continuer à tendre la main, à prendre et à manger de ses fruits. Bien plus, au milieu du dernier passage se trouve une parole du Seigneur encore plus énigmatique: «Voici, l'homme est devenu comme l'un de nous pour ce qui est de la connaissance du bien et du mal » (Gn 3,22b). Cette affirmation est souvent considérée comme ironique ou pour le moins hyperbolique. Toutefois, elle semble représenter une clé d'interprétation essentielle pour le texte. Le poids de cette affirmation du Seigneur repose évidemment tout entier sur le «comme ». Adam et Ève sont devenus " comme Dieu », dans le sens que, après avoir mangé de l'arbre, ils peuvent décider de ce qui est bien ou mal. Leur décision se situe, exactement comme celle $d u$ serpent $^{8}$, sur la ligne d'une sagesse différente et opposée à celle de Dieu, bien qu'elle ait quelque ressemblance avec sa nature, quant au libre arbitre. Wénin a noté que l'expression pourrait aussi être rendue par: "et vous serez comme des dieux ${ }^{9} . .$. ». Une telle traduction est certainement possible du point de vue grammatical, étant donné que le substantif 'êlōhîm, au pluriel, est utilisé dans l'Écriture pour indiquer aussi bien le «Dieu » de la révélation que les autres dieux. Il s'agit, probablement, d'un tartê mašma ("double signification »), expédient rabbinique fondé sur l'ambivalence sémantique d'un terme pour indiquer deux sens en même temps. Cela signifie que, voulant être « comme » Dieu, nos premiers 
parents sont en réalité devenus comme des dieux, tombant ainsi dans l'idolâtrie ${ }^{10}$. En outre, l'histoire du salut démontre, sans l'ombre d'un doute, que la voie d'accès à l'arbre de la connaissance du bien et du mal demeure ouverte. Chaque transgression dont l'homme est individuellement responsable suit, plus ou moins, le même parcours. En effet, dans tout péché l'homme transgresse un commandement de Dieu et, ce faisant, il s'approprie le droit de choisir ce qui est bien ou ce qui est mal. Dieu accepte la fragilité humaine et le péché, et continue à concéder à l'homme la possibilité de continuer à "se faire dieu de soi-même", à exercer son libre arbitre, mais il veut rendre manifeste que l'autonomie morale ${ }^{11}$, détachée de la volonté divine, implique pour l'homme l'impossibilité d'accéder à l'arbre de la vie.

7 Un autre aspect mérite aussi d'être relevé. Dans les premiers versets du premier passage, c'est la femme qui joue le rôle principal. C'est elle qui parle avec le serpent, alors qu'Adam n'apparaît même pas dans la scène. Dans le dernier passage au contraire c'est l'homme le protagoniste : il donne son nom à la femme et, après qu'il a reçu les tuniques de peau, le Seigneur semble ne s'adresser qu'à lui $\left(3,22-24^{12}\right)$. Ce fait revêt une très grande importance si on interprète le texte en le mettant en rapport avec $\mathrm{Gn}$ 2,16-17. En effet, le commandement de ne pas manger du fruit de l'arbre du bien et du mal lui aussi avait été adressé à l'homme. Ainsi l'auteur réussit à souligner de manière efficace la responsabilité d'Adam dans la faute, et donc une imputabilité majeure par rapport à Ève.

\section{Les passages intermédiaires $(6-8 ; 14-19)$}

\section{Composition}

8 Le deuxième passage comprend trois parties organisées de manière concentrique. Chacune commence avec un verbe de perception (« et il vit », 6a ; « et s'ouvrirent leurs yeux [...] et ils s'aperçurent », 7 ; « et ils entendirent », 8a), suivi d'une série d'actions qui, progressivement, tissent les fils de la narration. Tous les verbes sont à la forme narrative wayyiqtōl. La première scène rapporte le péché de nos premiers parents, la deuxième décrit la conséquence immédiate de cet acte malheureux, à savoir le sentiment de la nudité, la troisième enfin dit l'impact de la confrontation avec le Créateur. On peut noter en outre une correspondance globale entre les parties extrêmes. Dans la première Ève se trouve devant l'arbre, objet de sa passion, qui représente l'objet de l'interdit du commandement divin et elle pèche en entraînant aussi Adam dans la même transgression. Dans la dernière partie nos premiers parents se trouvent en présence de Yhwh et, de manière paradoxale et quelque peu grotesque, ils se cachent au milieu des arbres.

9 Le quatrième passage compte lui aussi trois parties $(14-15 ; 16.17-19)$. Chacune rapporte la sentence du Seigneur Dieu adressée aux trois personnages : le serpent, la femme et l'homme. Le parallélisme suit l'ordre chronologique. Le châtiment en effet suit l'ordre temporel des rébellions. En revanche, il est difficile de supposer un ordre de responsabilité par rapport à la faute, étant donné que le précepte avait été donne par Dieu à Adam et non à sa femme. La responsabilité de ce dernier apparaît, comme on l'a vu, plus grande de celle d'Ève. Du point de vue rhétorique, en effet, les parties extrêmes se correspondent: elles sont plus développées et contiennent toutes deux une malédiction («maudit») qui durera " pour tous les jours de la vie » du coupable (14.17). 
Y est indiqué aussi le "pourquoi » (14.17) de la sentence. De nouveau il s'agit d'une structure concentrique, ce qui établit une correspondance avec la structure du deuxième passage.

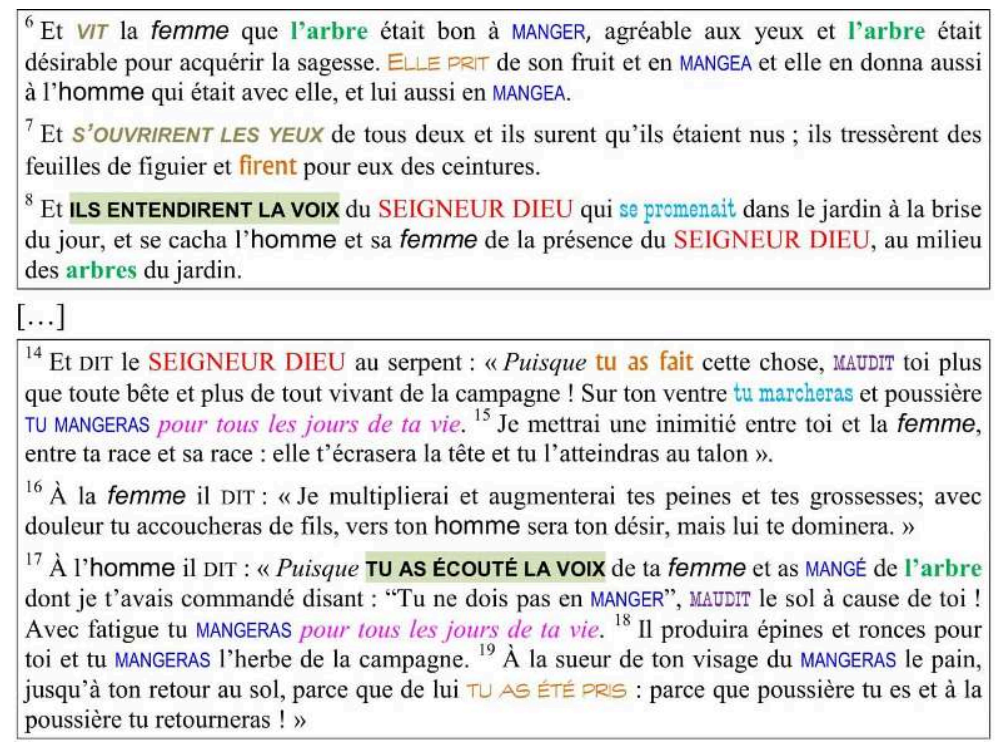

10 En outre, les passages intermédiaires se correspondent globalement. Y sont rapportés respectivement le péché et les conséquences de cet acte malheureux sur les coupables, à savoir la sentence du Seigneur. Le verbe "manger » est celui qui revient le plus souvent : neuf fois en tout : 6 (trois fois) ; 14.17 (quatre fois) ; 18.19 (deux fois). Il joue le rôle de termes initiaux $(6$, trois fois ; 14$)$ et de termes extrêmes, étant donné qu'il revient massivement aux extrémités (6, trois fois ; 17-19, cinq fois). On peut considérer que le même rôle est rempli aussi par le verbe " prendre » (6.19). Les termes « jour(s) », " se promener » / " marcher $^{13}$ » et l'expression "Seigneur Dieu » sont termes médians à distance (8.14). On pourra encore noter les reprises lexicales suivantes :

- « $\operatorname{arbre}(\mathrm{s}) »(6 b i s .8 ; 17)$;

- « homme » $(6.8 ; 14.16 .17)$ et « femme » $(6.8 ; 15.16 .17)$;

- « faire » $(7 ; 14)$;

- « écouter la voix » $(8 ; 17)$; cette expression établit un parallèle entre les deux voix : Adam aurait dû écouter la voix du Seigneur, alors qu'il a écouté celle de sa femme.

\section{Interprétation}

11 Dans le deuxième passage, comme on l'a vu pour le premier, ressort de nouveau le rôle de premier plan de la femme. L'acte qui précède et motive sa décision de manger du fruit est décrit de manière détaillée. Il semble presque que la scène s'attarde sur la contemplation du fruit défendu (6). La femme reste ravie et complètement prise par la tentation tandis que mûrit progressivement sa décision. Adam au contraire reste encore à l'arrière-plan. On ne raconte même pas comment il a été induit au péché. Le texte rapporte simplement qu'il reçoit le fruit défendu de la main d'Ève, sa femme, et qu'il le mange (6). Au contraire, son rôle commence à émerger quand apparaissent les conséquences du péché. Tous deux en effet se rendent compte qu'ils sont nus. Tous deux ensuite entendent la voix du Seigneur Dieu et se cachent. Cependant, affleure ici un détail fondamental: pour la première fois dans le récit, l'homme est mentionné avant la femme (8). Il faut noter en revanche le rôle fondamental d'Adam aux yeux de Dieu au moment du verdict. En effet, le jugement d'Adam 1) apparaît en 
correspondance symétrique à celui du serpent ; 2) il est plus développé que celui d'Ève ; 3) il contient une malédiction qui durera "pour tous les jours de sa vie» (17);4) il inclut une référence claire à la mort physique (« jusqu’à ton retour au sol»).

De la comparaison des passages intermédiaires ressort de nouveau l'importance de la responsabilité d'Adam, bien que ce soit plutôt Ève qui semble la protagoniste du récit. On peut ainsi penser que, dans le but que le serpent s'était fixé, le rôle de la femme était celui de faire tomber « Adam ».

Il vaut aussi la peine de souligner l'usage du verbe "manger ». Dans le deuxième passage, le péché consiste à "manger » le fruit interdit. Le terme se trouve trois fois dans le même verset (6). Dans le quatrième passage en revanche il n'est pas utilisé moins de six fois (14.17ter.18.19). Toutefois, la chose la plus remarquable est qu'aussi bien le serpent que l'homme sont frappés par les conséquences du péché en rapport avec l'objet de leur nourriture : l'un sera condamné à manger de la poussière tous les jours de sa vie (14), l'autre ne pourra manger qu'à la sueur de son front (17-18). Cela signifie probablement que le péché affaiblit la personne qui est engagée dans la désobéissance.

\section{L'ensemble de la séquence $(3,1-24)$}

\section{Composition}

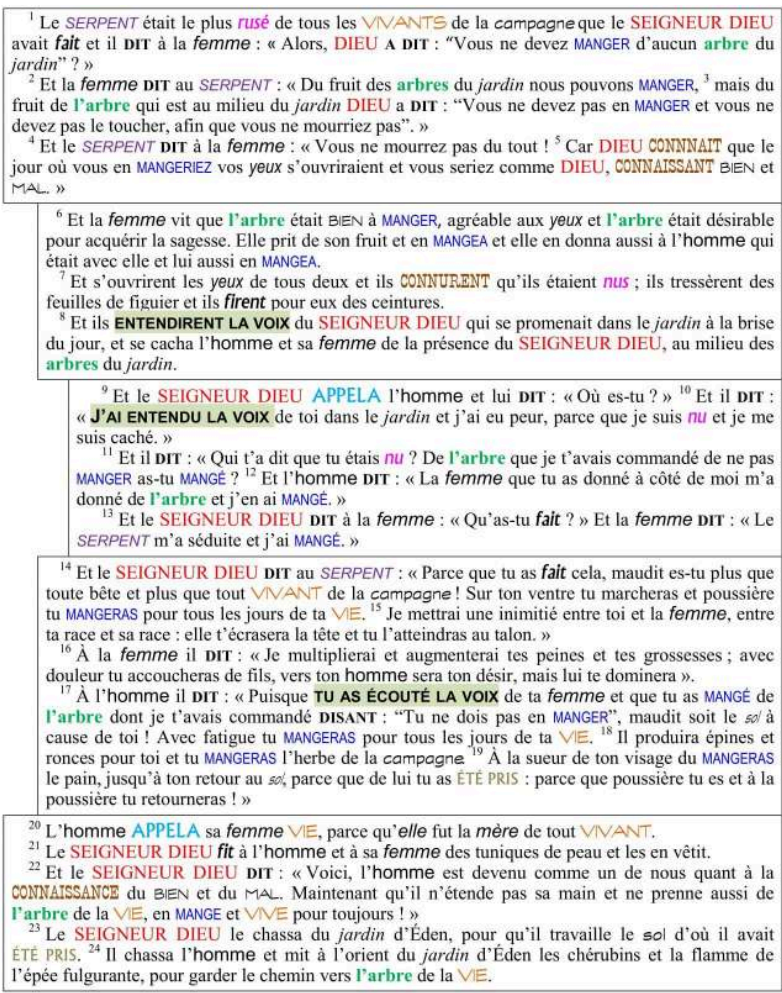

Le passage central (9-13) comprend trois parties $(9-10,11-12,13)$ organisées de manière concentrique. L'expression «et il dit» revient six fois, deux dans chaque partie (9.10.11.12.13bis). La partie centrale est plus développée que les autres et contient deux questions. Les parties extrêmes se correspondent : elles comportent la question initiale adressée à Dieu, à Adam, puis à Ève. 
15 Au centre de la séquence (9-13), comme il arrive souvent, des questions : quatre pour être précis (9b.11bis.13). Ce passage se caractérise par le rîb («controverse», "dispute ", "procès ») de Dieu qui met en lumière la transgression de nos premiers parents.

16 La séquence est marquée par de nombreuses reprises lexicales. Voici les plus significatives :

- «Manger » est le mot-clé de la séquence : il revient 17 fois $^{14}$ : dans les passages extrêmes et dans le passage central il revient 6 fois (2bis.11ter.22) avec «arbre » (2bis.11bis.22 $2^{15}$ ), et il indique le problème fondamental du récit de Gn 3 ;

- «Dieu » revient 13 fois, 9 fois accompagné du tétragramme sacré «Yhwh »; il remplit la fonction de terme initial 4 fois (1.9.14.21); il est terme médian entre les trois passages centraux $(8 b i s .9 ; 13.14)$, terme final en 4 bis (le seul terme « Dieu ») avec 8 bis et 13 ;

- « femme » est repris 13 fois, dont 2 comme termes initiaux ;

- « homme ${ }^{16}$ » revient 10 fois, dont 2 comme termes initiaux entre le troisième et le cinquième passage (9.20) avec le verbe « appeler» ;

- « serpent » est utilisé 5 fois, dont 2 comme termes initiaux;

- il faut noter qu'au début de chaque passage apparaît toujours un des quatre personnages principaux du récit, ce qui confirme la composition proposée ;

- l'expression "plus que tout vivant de la campagne » apparaît deux fois près du début d'un passage (1.14) ; elle est proche du syntagme «tout vivant » de 20 au début du dernier passage et des termes « vie/vivre » $(14.17 ; 20.22 .24)$;

- « écouter la voix », termes médians entre 8 et 9 , revient aussi en 17 ;

- « être tiré ${ }^{17}$ du sol ${ }^{18}$ » se trouve à la fin des deux derniers passages (19.23);

- l'adjectif « nu » revient 3 fois et il est terme central en 7 et 11 , en outre il rappelle « rusé » de $1^{19}$;

- le terme « œil » revient 3 fois, dont 2 en termes médians avec « bien/bon ».

\section{Interprétation}

\section{L'utilisation de l'expression « Seigneur Dieu »}

On a déjà noté que le terme «Dieu », accompagné du tétragramme sacré «Yhwh » et traduit ici par « Seigneur Dieu » revient en tout neuf fois (1.8bis.9.13.14.21.22.23), tandis qu'ailleurs il apparaît seul quatre fois. Il est remarquable que l'expression complète "Seigneur Dieu» soit toujours prononcée par le narrateur et jamais pas les autres interlocuteurs, serpent, homme et femme, tandis que le terme «Dieu» sans le tétragramme sacré est employé quatre fois, toutes dans le dialogue initial qui induit Ève à prendre du fruit de l'arbre interdit. Trois fois il est prononcé par le serpent (1.5bis) et une fois par la femme (2). Cela correspond probablement au fait que l'auteur a voulu souligner ainsi que dans le dialogue, aussi bien le serpent que la femme refusent de reconnaître la gloire de Dieu. Il est connu que le tétragramme est un qereketiv perpétuel et ne possède pas de voyelles propres, mais celles de 'ădōnāy, «mon Seigneur ». On peut donc penser que le fait de ne pas mentionner le nom sacré implique aussi le refus de sa seigneurie. Le serpent et Ève ne veulent pas se soumettre à l'autorité de Dieu sur l'histoire et sur le monde créé. Tout cela établit un contraste très fort avec les deux premiers chapitres de la Genèse où la création entière apparaît soumise à l'homme et ce dernier à son créateur (voir Gn 1,26.29-31;2,16-17.19-20).

\section{Les différentes étapes du rîb prophétique en Gn 3}

18 Il a déjà été question de la relation entre le jugement de Dieu et la figure vétérotestamentaire du rîb prophétique étudié par Pietro Bovati ${ }^{20}$. Il semble opportun de reprendre la question pour vérifier en détail en Gn 3 la présence ou non des éléments de la controverse juridique. 
19 Avant tout, le rîb est une controverse ou dispute de caractère judiciaire entre deux parties en rapport de type juridique. Il faut distinguer entre le rîb qui surgit entre parties égales et celui où une des deux parties est Yhwh. Dans ce dernier cas il est clair qu'il s'agit de l'alliance. Ce type de relations entre Yhwh et nos premiers parents sont établies par le commandement donné par Dieu à l'homme (2,16-17) après qu'il l'a créé et qu'il lui a donné toute une série de bienfaits, entre autres celui de vivre dans le jardin d'Éden.

$20 \mathrm{Au}$ début c'est normalement une situation de paix et d'accord, jusqu'au moment où survient un évènement qui produit un désordre ou un dérèglement du rapport qui peut aller jusqu'à détériorer un des éléments fondamentaux du pacte qui liait les parties. Une telle situation d'harmonie initiale peut se vérifier, dans notre cas, jusqu'à Gn 3,5, parce que la rupture formelle du commandement arrive seulement en 3,6.

21 Une fois que s'est produit le fait qui trouble le rapport, la partie lésée entreprend une action dont le but est de rétablir la justice. Nous nous trouvons ici dans le cadre spécifique du rî $b^{21}$. Quand l'action "judiciaire» est entreprise par Yhwh, il est des éléments fondamentaux qu'il est important de connaître. Le premier est que l'intention fondamentale d'une telle procédure est de reconduire le coupable dans la voie de la vérité et de la justice, c'est-à-dire sur le juste chemin. Il ne s'agit donc pas de disqualifier ou de supprimer le coupable. Le deuxième aspect est que le pardon précède l'action judiciaire, qu'il est un acte originaire, la condition sine qua non implicite dès le premier instant. L'acceptation pleine de l'action entreprise par la partie lésée et la reconnaissance de la faute ouvrent donc immédiatement la perspective de la réconciliation ${ }^{22}$. Le troisième aspect fondamental est que le rîb a aussi pour finalité de déclarer l'innocence de la partie lésée. Dans le cas de Gn 3 il est clair que le rîb de Dieu se trouve dans le passage central (9-13) qui, selon les lois de la rhétorique biblique, recèle aussi la clé d'interprétation de toute la séquence. Ici Yhwh convoque Adam et Ève et leur demande compte de ce qui s'est passé. Le lexique typique de cette procédure juridique confirme cette lecture. Quand Dieu cite Adam à comparaitre, le texte utilise la racine verbale $q r$ ', " appeler ", terme typique de la convocation émise par le juge. De même, la dénonciation du crime de la part de Yhwh, énoncée de manière interrogative, est marquée de nouveau par le lexique caractéristique du rîb. En effet, la première question adressée à Adam commence par 'ê, « où ? ( (9), tandis que celle qui est posée à Ève commence par mah $z \bar{o} t$, «qu'est $\operatorname{ceci}^{23}$ ? ». Une fois interrogés, Adam et Ève n'acceptent pas leur propre responsabilité et ne demandent pas pardon (voir 12.13b). Après le rîb, si l'accusé se repent, reconnait son erreur, demande pardon et éventuellement indemnise de manière adéquate la partie lésée, advient la réconciliation, qui débouche éventuellement sur un nouvel accord. Si au contraire l'accusé se déclare innocent ou trouve des justifications que la partie lésée n'accepte pas, il est alors traîné au tribunal ou un conflit éclatera entre les deux parties. Cependant, lorsque c'est Yhwh qui entreprend l'action judiciaire, il est clair qu'il n'est aucun tribunal terrestre qui ait le pouvoir de juger ${ }^{24}$, mais que c'est Dieu lui-même qui émet la sentence et la rend exécutoire. En Gn 3 ces deux derniers aspects se vérifient dans les deux derniers passages avec les sentences adressées aux trois coupables et l'expulsion de l'Éden (14-19;20-24).

22 Le lecteur attentif aura noté que dans chaque passage de la séquence se trouve un des moments de cette figure juridique vétérotestamentaire. Cela ne semble pas dû au hasard. Il est même possible de poser l'hypothèse que Gn 3 constitue le premier rîb de la 
Bible, le premier appel à la conversion. L'assimilation du récit de la chute de nos premiers parents à cette figure juridique ne doit pas étonner, car la façon d'agir de Dieu dans l'histoire est toujours la même. Au contraire, une telle constatation aide à mieux comprendre la finalité de l'action divine. Dieu n'apparaît pas dans l'histoire de nos premiers parents pour émettre une sentence et les chasser du jardin; il les cite à comparaître pour qu'ils reconnaissent leur faute, demandent pardon et reviennent à la communion avec leur créateur. C'est seulement après la faillite de cette action que Dieu prononce le verdict. Pour confirmer encore ce qui vient d'être dit, on notera qu'il se trouve dans la sentence même deux signes sans équivoque de l'amour de Dieu. Le premier est ce qu'on appelle le "protévangile", qui contient l'annonce de la victoire finale de la descendance de la femme sur celle du serpent (15). Le deuxième est le don des tuniques de peau, qui prend sens dans le langage sponsal de l'époque, c'est-à-dire dans les relations entre mari et épouse. Ce don signifie que Dieu, de son côté, n'a nullement l'intention de rompre l'alliance avec le premier couple, disposé qu'il est à pourvoir à leur vêtement, comme un mari le fait pour son épouse. L'expulsion du paradis ne fait rien d'autre alors que rendre visible ce qui était déjà advenu, à savoir la rupture de la relation avec Dieu de la part de l'homme. Dieu en revanche reste toujours fidèle. La sentence acquiert ainsi une valeur de remède et la souffrance est en même temps conséquence du péché et chemin de rédemption.

\section{La coïncidence des centres}

Un autre aspect important est la relation entre les centres des passages. Au cœur du second, «être nu» (7) est le premier acte de connaissance de nos premiers parents après qu'ils ont mangé du fruit de l'arbre interdit. Au centre du troisième passage au contraire se trouve l'indice sans équivoque que la transgression a été accomplie (11). Le texte établit une claire connexion entre l'aspiration d'Adam et Ève de manger, et donc, en quelque sorte, de posséder la connaissance du bien et du mal et la conséquence immédiate de cette possession. L'auteur veut déchiffrer et mettre en lumière sans plus tarder ce que provoque le fait de posséder et de se nourrir du fruit de l'arbre de l'autonomie morale : l'expérience de la nudité. Celle-ci est ici un acte cognitif intime de désorientation qui exprime embarras, malaise et confusion ${ }^{25}$. La scène est certainement différente de la scène idyllique de Gn 2,25, quand il est affirmé à l'aube de la création : " Or tous deux étaient nus, l'homme et sa femme, et ils n'avaient pas honte ». La honte maintenant semble prendre le dessus, même si le mot n'est pas utilisé. Mais le fait de se cacher parmi les arbres du jardin montre à l'évidence l'embarras. Le terme du texte massorétique pour indiquer la nudité de nos premiers parents est 'êrôm. Selon l'étude réalisée par Herbert Niehr, ce mot, à part le cas cité à l'instant (Gn 2,25), possède dans toutes ses autres occurrences dans l'Ancien Testament une connotation négative liée au sens de "pauvreté », de «deuil», d'«emprisonnement» et d'« adultère ». Ce dernier aspect revêt une importance notable. En effet, en différentes occasions être nu est le signe de la répudiation pour cause d'adultère. En ce cas, la nudité exprime d'une part la honte de la femme qui s'est souillée par l'adultère, d'autre part elle indique la cessation de l'obligation pour le mari de pourvoir à l'habillement de son épouse ${ }^{26}$. Les prophètes ont utilisé fréquemment l'image de la femme adultère qui est dépouillée pour dénoncer la honte du péché du peuple élu et la rupture de l'Alliance ${ }^{27}$. On peut donc penser que, dans le cas de Gn 3, l'idée de la nudité implique la honte d'être découverts par Dieu comme transgresseurs du précepte. 
dit ", comme on peut le voir dans la réécriture, ce qui attire l'attention sur le fait que Yhwh avait donné le commandement à Adam avant de le placer dans le jardin d'Éden (Gn 2,16-17). En comparant ce commandement avec les paroles du serpent et de la femme, apparaissait clairement que ni l'un ni l'autre ne rapportait correctement ce qu'en réalité Dieu avait ordonné.

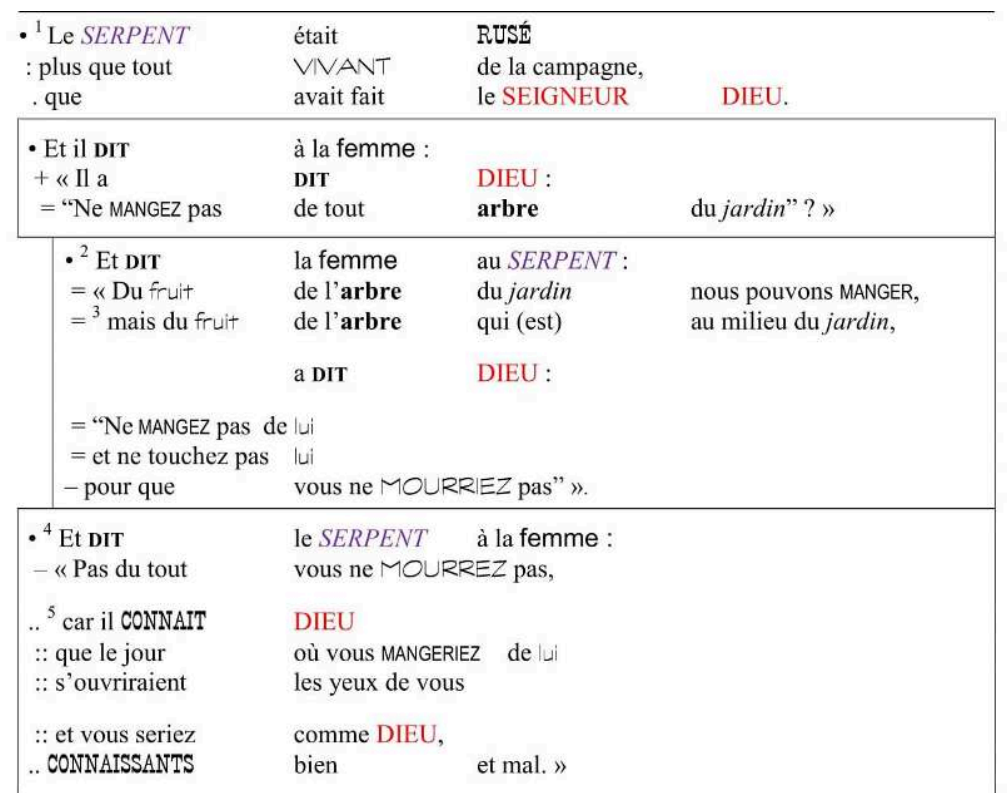

Le serpent présente seulement la partie négative du précepte, la défense de manger d'un arbre déterminé, déplaçant toute l'attention sur la limite imposée par Dieu et obscurcissant ainsi l'ample espace de liberté concédé à nos premiers parents (« de tous les arbres tu pourras manger $»)$. En outre, le tentateur introduit dans sa formulation plusieurs mensonges. L'un est très facile à découvrir : "Alors, Dieu a dit: "Vous ne mangerez pas de tous les arbres du jardin?» (1). D'autres sont beaucoup plus subtils, comme, par exemple, le fait que dans la même question le commandement soit relativisé (voir le "alors ${ }^{29}$ ») et qu'il omette de mentionner les conséquences du péché incluses dans le précepte divin (« certainement vous mourrez »; 2,17), ce qu'il niera au moment opportun : «Pas du tout, vous ne mourrez pas » $(3,4)$.

Pour sa part, la femme aussi déforme la formulation du commandement divin : elle ajoute quelque chose que le Seigneur Dieu n'avait pas dit, rendant le précepte plus rigide (« n'en mangez pas et n'y touchez pas »: 3,3 ); quand elle parle des conséquences du péché, elle omet le « certainement » et elle confond l'arbre de la vie avec celui de la connaissance du bien et du mal ${ }^{30}$. Une telle confusion pourrait signifier que l'arbre interdit, celui du bien et du mal, semble être le véritable arbre de la vie, celui qui donne le bonheur ${ }^{31}$. Le résultat final est que tous discutent du précepte, mais en réalité personne ne dit réellement ce que Dieu a commandé, de sorte qu'Ève s'enlise lentement dans les sables mouvants de la tentation. Or, le fait pertinent est qu'au cœur de la 
séquence (11) se trouve de nouveau le commandement, mais cette fois prononcé par Dieu lui-même.

\section{Le centre de la séquence}

Dans le passage central, Yhwh cite l'homme à comparaitre, l'interroge en premier et lui pose trois questions (9.11), dont deux occupent le centre de la composition (11). Dans ce dialogue, la femme reste à l'arrière-plan et elle est interpellée en dernier, tandis qu'Adam est l'imputé principal. En effet, c'est à lui que Dieu demande compte du commandement. L'expression "écouter la voix", qui joue le rôle de termes médians entre le deuxième passage et le passage central, rappelle clairement quelle était la mission primordiale de l'homme : écouter le commandement de Dieu pour le garder (2,16-17). En revanche, dans la cause de la sentence affleure le dilemme essentiel: Adam a préféré écouter la voix de la femme. Le problème se trouve donc dans l'écoute, tant que le fait d'entendre la voix du Seigneur devient occasion de peur (10). Il est clair que l'action de Dieu est mue par le désir d'appeler nos premiers parents à la conversion. Il n'abandonne pas sa créature, mais veut la pousser à reconnaître sa propre responsabilité. Adam et Ève au contraire restent inflexibles sur leur position, ils perçoivent l'action de Dieu comme une menace pour leur intégrité (10) et ils refusent de reconnaître leur faute (12-13).

Le commandement se trouve de nouveau au milieu d'un passage, le troisième, et il est prononcé par Dieu lui-même. Il vaut la peine, encore une fois, de reprendre le cadre de ce passage, analysé dans une contribution précédente.

Le cœur du passage central contient la formulation exacte du précepte, même si c'est de manière synthétique. Cela signifie que le commandement est remis en lumière pour corriger les mensonges contenus dans la formulation du serpent (1) et les erreurs dans la formulation de la femme (2). Étrangement, le Seigneur aussi omet de rappeler les conséquences immédiates du péché ("certainement vous mourrez ») : il ne s'agit pas d'une inattention de l'auteur et encore moins de Dieu. Du point de vue rhétorique on peut penser qu'il s'agit d'un procédé littéraire qui, de manière énigmatique, a pour but de souligner l'importance de la question précédente : "Qui t'a appris que tu es nu?». La nudité, en effet, comme on l'a vu, pourrait impliquer la honte de se voir découverts par Dieu comme transgresseurs du précepte, ainsi que la désorientation spirituelle, conséquence immédiate du péché.

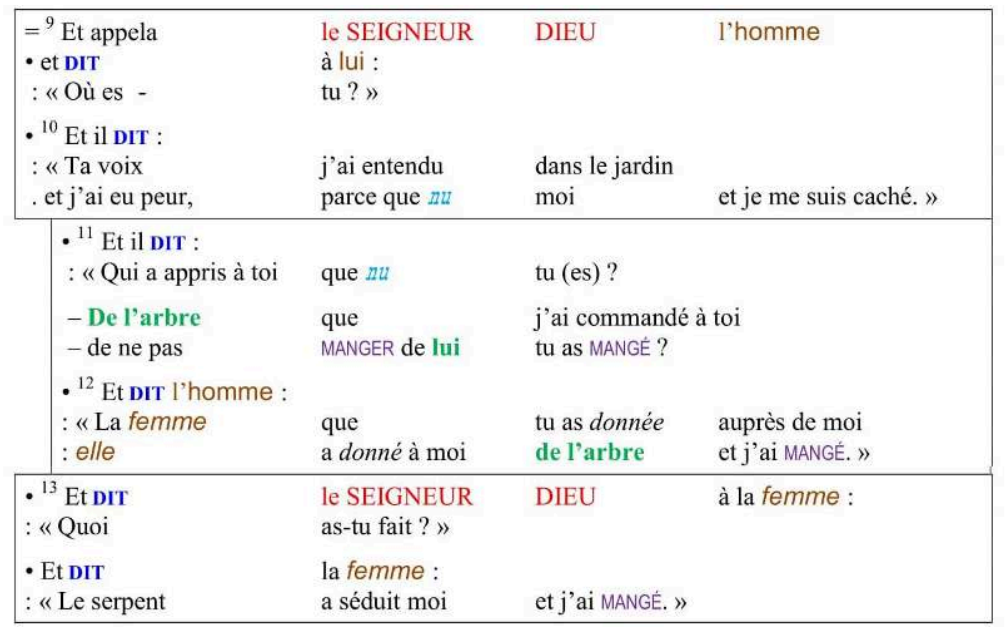


31 À la lumière du rîb, dont il a été question précédemment, on peut comprendre maintenant le rôle de clé de voûte que joue le centre pour toute la séquence. En effet, c'est là qu'advient proprement l'appel à la conversion et c'est là que l'homme refuse d'admettre sa propre culpabilité en accusant la femme. Après quoi Ève fait retomber la faute sur le serpent et la justice n'est pas manifestée. Il n'y a ni reconnaissance de la faute, ni conversion, ni demande de pardon. La clé de compréhension de tout Gn 3 se trouve justement là. Tout ce qui suit n'est autre que la conséquence de l'endurcissement de nos premiers parents. Maintenant la sentence de Yhwh et l'expulsion de l'Éden acquièrent une lumière nouvelle.

\section{Conclusion}

32 L'analyse rhétorique de Gn 3 a aidé à éclairer certains points essentiels du récit de la chute de nos premiers parents. Le texte souligne avant tout la responsabilité d'Adam, égale ou probablement supérieure à celle d'Ève, ce qui ne ressort pas à une lecture superficielle du récit, où la femme semble jouer le rôle principal. En second lieu, on découvre graduellement l'intention profonde de l'agir de Dieu. Il ne vient pas pour châtier ni pour condamner Adam et $\mathrm{Eve}^{32}$, mais il leur offre la possibilité de la conversion, qui s'exprime à travers la forme juridico-littéraire du rîb. C'est seulement après avoir constaté l'endurcissement et la fuite de la responsabilité, qu'il ne lui reste d'autre alternative que d'offrir une deuxième voie de salut qui permettra à l'humain de s'engager sur un chemin de conversion en dehors de l'Éden. La sentence et son exécution ont pour finalité primordiale de laisser l'homme parcourir jusqu'au bout les conséquences de son propre choix, jusqu'à la reconnaissance de ses propres responsabilités. C'est seulement quand ils buteront contre leur propre limite, contre les adversités et les souffrances de la vie, que l'homme et la femme pourront reconnaître petit à petit leur réalité et se convertir. La sentence, avec tout ce qu'elle implique, son exécution et la souffrance, deviennent ainsi un remède pour le salut annoncé, de manière paradoxale, à l'intérieur même du verdict adressé au serpent, dans le fameux protévangile. La conséquence du péché devient en même temps chemin de rachat, ultérieure possibilité de revenir à Dieu.

Cette "seconde possibilité » sera donnée, par la suite, au peuple d'Israël après le terrible péché d'idolâtrie du veau d'or et ses tragiques conséquences (Ex 34). C'est à cette lumière que doivent être aussi interprétés plusieurs passages du Nouveau Testament, par exemple, l'épisode de la femme adultère à laquelle Jésus donne une nouvelle possibilité de salut. Le geste mystérieux de Jésus, répété, d'écrire par terre avec le doigt, sur l'interprétation duquel les exégètes de tous les temps se sont penchés, peut être interprété en ce sens : de même que Dieu a donné une deuxième voie de salut à Adam et Ève et l'a offerte aussi au peuple d'Israël au moment de l'alliance au Sinaï, en écrivant une deuxième fois les tables de la loi, ainsi fait-il aussi avec la femme adultère. L'expulsion d'Adam et d'Ève hors de l'Éden et l'histoire qui suivra, bien que difficile et pleine d'épines, devient ainsi, comme celle de tout homme et de toute femme dont nos premiers parents sont le paradigme, espace de rédemption, remède au péché, « deuxième table de salut ».

34 L'humain est ainsi invité à reconnaître sa propre faute, qui équivaut à confesser la justice de Dieu: «Pour que tu montres ta justice quand tu parles, et que paraisse ta victoire quand tu juges » (Ps 51,6b). Il s'agit du chemin inverse par rapport à celui que 
parcoururent Adam et Ève, qui ouvre une solution au rîb entrepris par Dieu. Ainsi, le rîb, bien loin d'être un simple procédé littéraire, devient un modèle théologique de l'agir salvifique de Dieu.

\section{NOTES}

1. Le texte de référence est R. Meynet, Traité de rhétorique biblique, Paris, Lethielleux (Rhétorique Sémitique 4.11), 2007 ; Pendé, Gabalda, 2011.

2. G. Lori - F. G. Voltaggio, «"E sarete come Dio". Analisi retorica di Gen 3,1-8 », dans R. Meynet J. Oniszczuk éd., Studi del quarto convegno RBS. International Studies on Biblical and Semitic rhetoric, Rome, G\&B Press (Retorica Biblica e Semitica 4), 2015, p. 31-55.

3. Dans « Genesis 2:4b-3:24 : A Synchronic Approach »Journal of Biblical Literature 96 (1977), p. 161-177, J. T. Walsh propose une structure semblable.

Dans son étude synchronique toutefois il élimine les versets 20-21. De même A. Wénin accorde peu d'importance à ces versets et c'est pourquoi il considère 3,1-13 comme une composition unitaire concentrique (il propose en fait deux structures possibles), tandis que la sentence de Dieu (14-19) et la conclusion du récit (22-24) avec l'expulsion d'Adam et Ève de l'Éden semblent être isolées du reste (A. Wénin,

D'Adam à Abraham ou les errances de l'humain. Lecture de Genèse 1,1-12,4

, Paris, Le Cerf, coll. Lire la Bible, 2007, p. 90-93).

4. Noter l'opposition entre les centres des deux passages.

5. Ce terme renvoie au terme correspondant « homme » (3,20.21.22.23).

6. Le terme se retrouve aussi en 3,2 .

7. Le terme se retrouve aussi en 3,1.4.

8. Le texte s'ouvre sur une affirmation introductive de grande importance : « Le serpent était le plus rusé de tous les êtres vivants de la campagne » (Gn 3,1). Pour indiquer le caractère rusé du serpent, le texte massorétique utilise le terme 'ārûm, traduit justement par « rusé ». Toutefois les anciennes versions et les targums, sauf la Vulgate, le traduisent avec une autre nuance. Le Targum Neofiti et la Septante le rendent par «le plus intelligent» ou «le plus prudent ». Très intéressante est la traduction du TPsJ (Targum du pseudo Jonathan) qui dit : « le plus intelligent pour le mal ». En outre, le terme est en rapport avec «nu », utilisé en 2,25 et 3,7, et les deux termes font un jeu de mots. Un tel rapport semble insinuer que nos premiers parents sont devenus «nus comme le serpent - comme le dieu auquel ils ont cru» (A. Wénin, D'Adam à Abrahamoules errances de l'humain,op. cit., p. 107) ;voir aussi H. Niehr, "'ārôm / 'êrôm ", Grande Lessico dell'Antico Testamento, VI, p. 1035-1036; F. Festorazzi, La Bibbia e il problema delle origini. L'inizio della storia della salvezza, Brescia, Paideia (Esegesi Biblica 3), 1966, p. 109. Enfin, il ne faut pas oublier que le mot 'ārûm peut aussi avoir le sens positif de « prudent » (voir, par ex., Pr $12,16 ; 13,16)$. La sagesse du serpent est donc différente et opposée à celle de Dieu.

9. A. Wénin,D'Adam à Abraham,op. cit., p. 88.

10. En tout péché se cache une idolâtrie. Voir P. Beauchamp, D'une montagne à l'autre, la Loi de Dieu, Paris, Le Seuil, 1999, p. 46.

11. Voir A. Wénin, L'homme biblique. Lectures dans le premier Testament, Paris, Le Cerf, 2004, p. 51 ; id., «Le précepte d'Adonaï Dieu en Genèse 2,16-17. Narration et anthropologie », Revue de Science Religieuse 82 (2008), p. 306-307. 
12. Il ne s'agit pas pour autant de nier une interprétation collective, c'est-à-dire nier qu'ici par « homme » il faille entendre aussi toute l'humanité.

13. Ils sont tous deux de la racine hlk.

14. Le lecteur attentif aura compté 18 occurrences; toutefois en 6 la première des trois occurrences du terme "manger » est en réalité un substantif de la même racine 'kl qui pourrait être traduit par « nourriture ».

15. Ce sont des termes centraux.

16. Huit fois ce mot traduit 'ādām et 'î̌ deux fois (6.16). Bien que la différence n'apparaisse pas dans la traduction, il s'agit de deux termes semblables.

17. Littéralement « être pris ", de la racine lqh, qui se retrouve aussi en 6 et 22.

18. Le terme « sol » revient aussi en 17 .

19. Voir supra la note 8.

20. Voir P. Bovati, Ristabilire la giustizia. Procedure, vocabolario, orientamenti, Rome, Pontificio Istituto Biblico (Analecta Biblica 110), 1986, p. 21-148.

21. Selon M. De Roche ("Yahweh's Rîb Against Israel : A Reassessment of the so-called "Prophetic Lawsuit" in the Preexilic Prophets ", Journal of Biblical Literature 102 [1983], p. 568), le terme rîb ne signifie pas seulement en lui-même un processus judiciaire, mais indique de manière plus générale qu'une partie a une revendication contre une autre: entre autres exemples, il cite justement Gn 3,7-9.

22. Voir P. Bovati, Ristabilire la giustizia, op. cit., p. $22-23$; 67-70;140-143.

23. Voir ibid., p. 63-64; 203-204.

24. Voir ibid., p. 25.

25. Même si le terme « mort » n'apparaît pas, la scène est dominée par la réalité de la mort. Voir P. Beauchamp, Cinquante portraits bibliques, Paris, Le Seuil, 2000, p. 146.

26. H. Niehr, «'ārôm / 'êrôm», Grande Lessico dell'Antico Testamento, VI, p. 1039-1040.

27. Voir, par ex., Os 2,5 ; Jr 13,25-27 ; Ez 16,36-37 ; Na 3,5-7.

28. G. Lori - F. G. Voltaggio, «"E sarete come Dio" », op. cit., p. 32-33.

29. Dieu avait donné un ordre; en disant "Alors, Dieu a donné...», le serpent relativise le commandement, insinuant un doute.

30. Voir G. Lori - F. G. Voltaggio, " "E sarete come Dio" ", op. cit., p. 33-34 et 42-43 ; A. Wénin, L'homme biblique, op. cit., p. 51-52 et, du même auteur, D'Adam à Abraham,op. cit., p. 95-103.

31. Selon une autre interprétation, elle aussi très intéressante, la confusion des deux arbres indiquerait que, aux yeux de la femme, l'interdit occupe, au centre du jardin, la place de l'arbre de la vie. A. Wénin, D’Adam à Abraham,op. cit., p. 99.

32. Dieu n'est donc pas du tout un rival de l'homme, comme le serpent l'a fait croire à Adam et Ève avec ses mensonges ; selon A. Wénin (L’homme biblique, op. cit., p. 53), c'est là précisément que se situe le péché de l'homme: avoir vu en Dieu un rival. Voir aussi, du même auteur, «Le précepte d'Adonaï Dieu », op. cit., p. 306-307.

\section{AUTEURS}

\section{GERMANO LORI}

Professeur à la Domus Galileae en Israël, membre de la RBS 
FRANCESCO GIOSUÈ VOLTAGGIO

Professeur à la Domus Galileae en Israël 